\title{
Traffic Offloading through Wi-Fi Networks
}

\author{
Kritika Kapoor \\ Assitant Professor \\ Tramiet, Mandi
}

\begin{abstract}
Cell systems are as of now confronting movement overburden issue because of extreme developing interest of advanced mobile phones, tablets and portable PCs. The after effect of which top of the line gadgets twofold their information movement consistently and this pattern is relied upon to proceed with given the fast advancement of versatile social applications. This expansion in information movement is gauge to quicken and after effect of which they brought on a prompt requirement for offloading activity for ideal execution of both voice and information administrations. Subsequently, extraordinary inventive arrangements have risen to oversee information movement. A financially savvy and one of sensible arrangement is to offload cell movement through Wi-Fi, Femtocells or Delay Tolerant System (DTN) to decrease the weight on the cell organizes and furthermore increment the quality of service (QoS). Wi-Fi offloading is a procedure for tending to the portable information blast issue. Offloading portable information activity through pioneering correspondences is answering for tackle this issue. The thought is that the specialist organizations convey data over cell systems to just clients in the objective set.
\end{abstract}

\section{Keywords}

Congestion, Wi-Fi offloading, 3G, Target Users

\section{INTRODUCTION}

With the emergence of the smart devices which are wholly dominating the market in which there are multiple applications which are used like Facebook, Google plus, Weather forecasting are chiefly dominated by data. These applications consume higher bandwidth and large amount of data to process. In this way the network become more congested and organize operators get ready for offloading the information and its flag movement to work in approaches to enhance their financial aspects [2]. An immense capital speculation is required to have high transfer speed and fast information, so to cope from this issue operators have taken a way path turn by utilizing options utilizing little cell as Wi-Fi, Femtocells and so on. This option can effectively deal with the developing portable information activity [3]. As per report of Cisco that versatile information movement is relied upon to develop to 30.6 Additional bytes for every month by 2020 , an eightfold increment more than 2015. Versatile information movement will develop at a CAGR of 43 percent from 2016 to 2021 [1]. Among this movement, around $67 \%$ activity is because of portable video information. This remarkable increment in information activity is making challenges for existing cell systems.

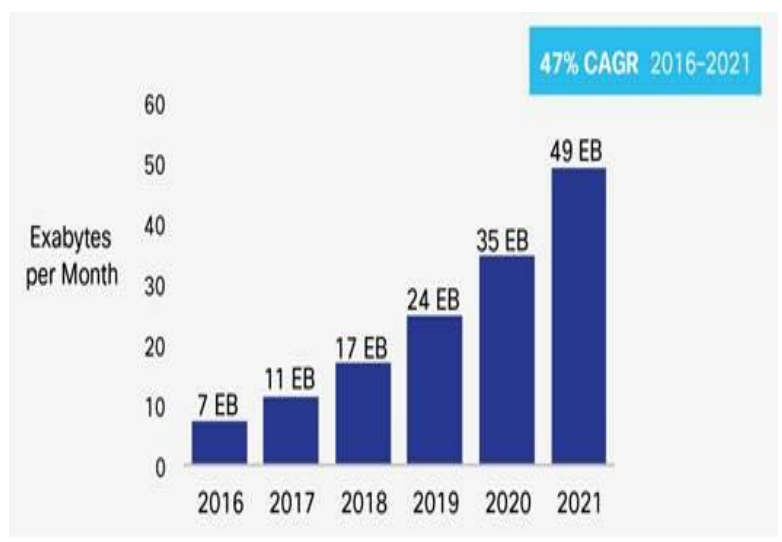

Figure 1: Global Mobile Data Traffic [1]

To handle this problem of explosive traffic demands growth and limited capacity of network there is urgent need to provide some better solution to reduce the traffic in the network. Various solutions has been provided to overcome this traffic like installing new base stations (BS's), increase the capacity of cellular networks by adding smaller cell size base stations, and also upgrading the cellular network to next generation advanced networks like Wi-Max or Long Term Evolution (LTE) [4]. This steep growth in traffic has put immense pressure on the traffic and the use of resources. After cellular technology, 3G technology came into existence but was not adaptable to support today mobile internet services. The theory of data offloading started in 2005 when the known name Unlicensed Mobile Access (UMA) came into existence which remained planned to allow contact to GSM and GPRS movable facilities [2] [3][19] [20].

Wi-Fi Offloading is method to naturally move activity from the cellular network to the neighborhood and diminishes the blockage in the network and improves utilization of accessible system assets. It is a standout amongst the most sparing and promising arrangements. The goal is to keep up nature of administration for client, while additionally diminishing the cost and effect of conveying limit hungry administrations on the portable network [5].

The mobile offloading action can be either done by end-user or network operator [6]. It empowers arrange operators to decrease the clog in the cell systems, while for the end-client it gives cost investment funds on information administrations and higher data transmission accessibility. By offloading portable information movement onto accessible correlative systems, operators can improve the accessible system assets and reduce the bottleneck of operators there by designating their system assets to different client.

There are two types of Wi-Fi offloading immediate offloading and deferred offloading [7] [16]. Immediate offloading is to utilize unconstrained availability to $\mathrm{Wi}-\mathrm{Fi}$ and exchange information on the spot. At the point when a client lets well enough alone for Wi-Fi scope, they suspend the offloading and all the incomplete exchanges are transmitted through cell systems. The vast majority of the advanced mobile phones as 
of now offer need to Wi-Fi over the cell interface in information transmissions can be required to right now accomplish the on-the-spot offloading. Deferred Offloading in this every information exchange is associated with a due date and it over and over resumes information exchange until the exchange is finished .These delay may happen because of the $\mathrm{Wi}-\mathrm{Fi}$ association issues or client in and out from the Wi-Fi scope region. On the off chance that information exchange does not complete inside its due date, cell organizes at long last total the exchange.

In this paper, we study the target-set selection problem as the first step toward mobile data offloading for information delivery. Target-users can help to further propagate the information among all the subscribed users through their social participation, when their mobile phones are within the transmission range of each other and can communicate opportunistically. Non target-users can also disseminate the information after they get it from target-users or others. The major advantage of this mobile data offloading approach is that there is almost no monetary cost associated with opportunistic communications. We investigate how to choose the initial target set with only $\mathrm{k}$ users, such that we can minimize the amount of mobile data traffic using game theory and SVM then different parameters has been evaluated.

This paper is organized as follows: We briefly review related work in Section 2. We then formulate the problem in Section 3. In Section 4, propose algorithms for the target-set selection Problem. In Section 5, we evaluate the performance by proposed algorithms and then conclusion and related work is discussed.

\section{RELATED WORK}

The number of researchers and practitioners has worked on analysis of wireless network and various studies have been made on mobile data traffic. To reduce the traffic in cellular network Wi-Fi offloading designs be created to deal with such voluminous movement. A few strategy and calculations have been acquainted over past numerous years with improve the activity over-burdening circumstance utilizing Wi-Fi offloading which empowers the reduce the measure of movement In this area, brief about Wi-Fi activity offloading that have been presented as key innovation in the course of the most recent couple of years. S. Ioannidis et al. [17] propose pushing updates of element substance from the framework to end client. They accept that the cell foundation has a settled total data transfer capacity that should be designated between end-client.

Y. Li et al. [18] build up a scientific system to ponder DTNbased versatile movement offloading of numerous portable information things and consider the ideal subset choice as a utility boost issue under various straight requirements, for example, activity heterogeneity, client portability. Chuang et al. [19] propose how to distribute content information to only target set of client via cellular networks and allow these target set user to further propagate the information through opportunistic communications. They basically proposed how to select initial target set client based on community based selection and in these communities select the initial source. Han et al. [20] implement offloading problem by subset determination instrument in light of client contact design. To endeavor Delay tolerant systems (DTNs) to encourage information scattering among versatile clients, to decrease the general cell activity they give three calculations. They propose how to select initial target set user to offload the mobile data traffic and how to minimize the amount of data traffic and also proposed opportunistic communication to forward information in the Mobile Social Networks.

Baier et al. [21]propose three particular estimations for separating advancement desires and present a computation, which uses these estimations to utilize a capable spearheading development offloading. They used approach of subset decision issue by envisioning the improvement of endcustomers with a particular true objective to gage future between contraption accessibility. Barbera et al. [23] investigate the contact design between end-clients, so as to choose a subset of focal VIP clients that are essential for the system as far as centrality and page-rank. In this they propose VIP designation, an answer for issue in view of the innate social parts of client versatility. X. Wang et al. [24] propose the framework of Traffic Offloading assisted by Social network services (SNS) via opportunistic Sharing in mobile social networks. TOSS pushes the content object to a properly selected group of seed client, who will opportunistically meet and share the content with others, depending on their spreading Impact in the SNS and their mobility impact in the MSN and share the content via opportunistic local connectivity. G. Gao et al. [25] focus on the problem of offloading many deadline-sensitive data items to some Wi-Fi APs with capacity constraints; that is, how to schedule each data item to the Wi-Fi APs, so that they can offload as many data items before their deadlines as possible, while taking the constraints of transmission capacity into consideration.

X. Zhuo et al. [26] mainly focused on how to stimulate client to offload cellular traffic via DTNs. They proposed an incentive framework for downlink mobile traffic offloading based on an auction mechanism, where client send bids, which include the delay it can tolerate and the discount the user wants to obtain for that delay, and a single provider buys the delay tolerance from the client. J. Lee et al. [27] studied how much economic benefits can be generated due to delayed Wi-Fi offloading, by modelling the interaction between a single provider and client based on a two-stage sequential game. X. Zhuo et al. [28] investigated the trade-off relationship between the amount of traffic being offloaded and the user's delay. They provided an incentive framework to motivate client to offload. They developed an incentive framework to motivate client to leverage their delay tolerance for cellular traffic offloading. B. Jung et al. [29] describe that heterogeneous network is responsible for collecting the network information, and decides the specific portion of traffic to be transmitted via $\mathrm{Wi}-\mathrm{Fi}$, to maximize the per-user throughput. Their purpose is to minimize the total transmission cost from the perspective of mobile client and take the deadline constraints. L. Gao et al. [30] analyse the interaction among one MNO and multiple APOs by using the Nash bargaining theory.

\section{PROBLEM STATEMENT}

Social media is one of the strongest platforms in these days for the people to be connected. Millions and millions of people share, post and comment at the social media. With the increasing number of client on social media, it also becomes very important to check whom a user is getting connected. There are several algorithms who work for the normalization of the client profile and data like greedy algorithm, heuristic algorithms etc. All these algorithms choose a target set through which the client is connected. The problem of this research work is to optimize the neutrality of the connected user through the target set only $\mathrm{z}$ no of users by using the game theory so that least power is consumed in the processing the data. This $\mathrm{z}$ number of users will decrease the traffic in the 
network. If there are total $\mathrm{x}$ no of connected users and among them only y users before deadline receive the data then traffic reduced is

$\mathbf{x}-(\mathbf{z}+(\mathbf{x}-\mathbf{y}))=\mathbf{y}-\mathbf{z}$

The following parameters are going to be evaluated at the end of the processing

a) Battery consumed at different hours of processing

b) Signal to Noise ratio

c) Mean Square Error

More specifically, the contribution of the paper is

- We used greedy algorithm to find the initial target set in which target set in find out by finding the degree of the nodes.

- Then cluster formation is made so that they take less time to propagate the information to rest of the users.

- Parameters have been evaluated by using support vector machine.

\section{PROPOSED APPROACH}

Initially we have set of users who are communicated to each other and connection is being created between these users. Using the dataset greedy and heuristic approach is applied and initial target set is found out. Firstly degree of all users is found by using some rule set and cluster formation is made depending upon the degree. The following advantages can be listed down:
1) Normalization of the clustering
2) Reduction of noisy nodes
3) Better efficiency
4) Less mean square error
5) Higher strength of communication
6) Less consumption of battery

In this some noisy nodes are also included these are the nodes which are required to neutralize the group but they do not belong to the cluster at the initial phase. Cluster formation and neutralization is done by using support vector machine in which node degree value and group information is provided. In this two functions SVM classify and SVM train used. They are basically used to find which nodes present in which cluster and their corresponding values. So that information is propagating in less time to maximum number of nodes. The following parameters have been evaluated:

1. Battery Consumed: A total of 5 slabs have been evaluated. Once the battery of the device is charged, it is evaluated after $1 \frac{1}{2}$ hour, 1 hour, 2 hours, 3 hours and 6 hours.

2. MSE: MSE stands for mean square error and mathematically it is defined as follows:

MSE $=(($ noisy group strength $)-($ non- noisy group Strength)) / (Total element in one group).

3. SNR: SNR stands for Signal to noise ratio and it evaluates the total randomness in a group against the total group strength. Mathematically, it can be defined as follows

$$
S N R=(\text { Max bit value } 2) / M S E
$$

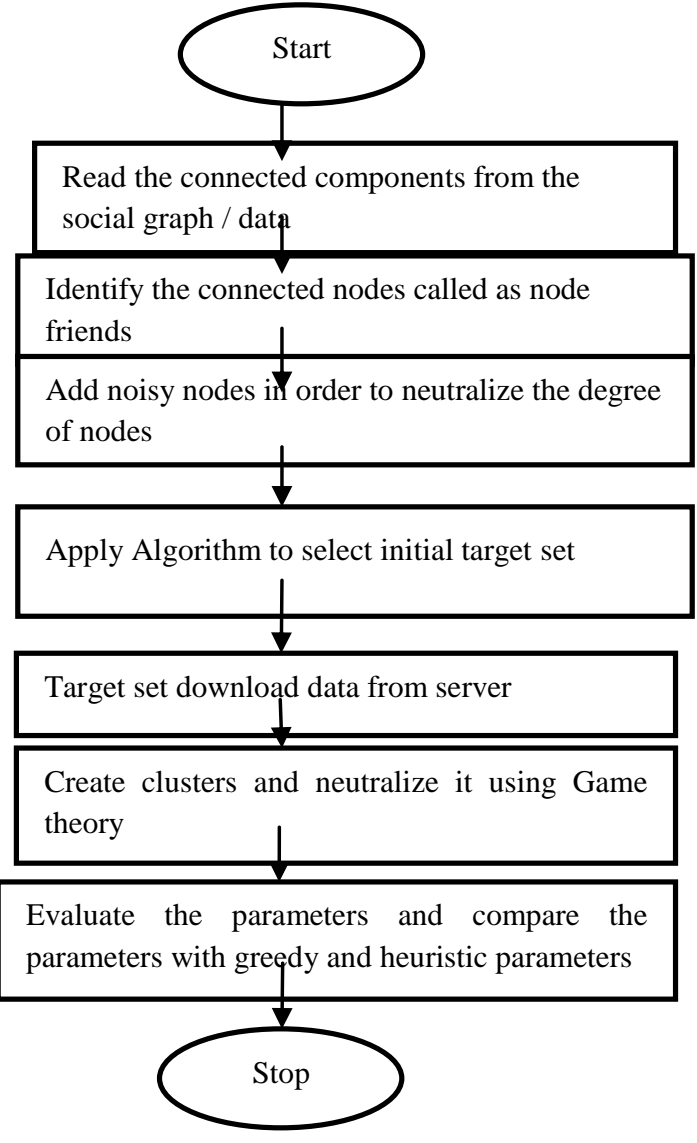

\section{RESULT AND ANALYSIS}

The neutralization has to be performed on these clients only by using greedy and heuristic approach. Firstly, the degree is find out for those who have same degree and must lying on same cluster and the remaining lied on different cluster then multi-SVM and greedy is applied to find out the other clients.

Table 1: Analysis

\begin{tabular}{|l|l|}
\hline Total no of nodes & 786 \\
\hline Target Selected & 38 \\
\hline Range & 250 meter \\
\hline Area & $1000^{*} 1000$ \\
\hline Physical Layer & Bluetooth, Wi-Fi \\
& \\
\hline
\end{tabular}

Then different parameters are evaluated like battery consumption, target set, Signal Noise Ratio, Mean Square Error. 
Table 2: Battery Consumption

\begin{tabular}{|c|c|c|}
\hline $\begin{array}{c}\text { Battery } \\
\text { Consumption } \\
\text { Bluetooth }\end{array}$ & $\begin{array}{c}\text { Battery } \\
\text { Consumption } \\
\text { Wi-Fi }\end{array}$ & $\begin{array}{c}\text { Battery } \\
\text { Consumption Wi- } \\
\text { Fi sleep }\end{array}$ \\
\hline 70.34272 & 3.092963 & 30.86594 \\
\hline 70.43231 & 2.184556 & 30.64314 \\
\hline 70.49282 & 2.168105 & 30.39878 \\
\hline 70.53174 & 3.949731 & 30.15915 \\
\hline 70.47921 & 3.941977 & 30.57173 \\
\hline 70.50166 & 3.65894 & 30.80652 \\
\hline 70.96901 & 2.39671 & 30.71554 \\
\hline
\end{tabular}

The table clearly depicts that Battery Consumption over bluetooth is maximum where as the battery consumption over wifi is least. This is because that the wifi usages is minimal . The the wifi is in sleep mode, it takes additional data from other devices and hence uses more battery as compared to the wifi normal but obviously less than than of the bluetooth. The bluetooth connection cosumes a lot of energy as it uses the internal power of the device. The differnce in percentage computation is more than $100 \%$ between the wifi and the bluetooth.

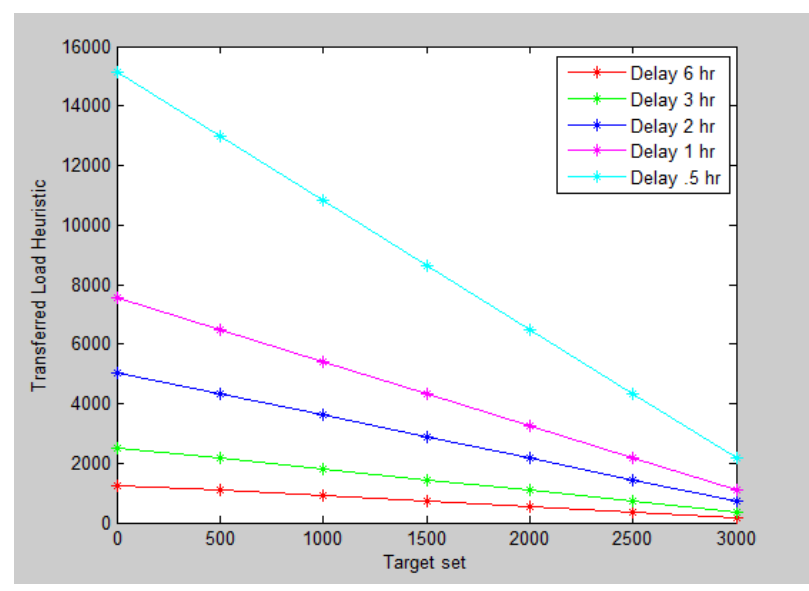

Figure 2: Target set with transferred load

The above figure depicts the transferred load against different target sets. In the figure the $\mathrm{x}$ axis denote the target set and $\mathrm{y}$ axis denote transfer load heuristic. Above mentioned figure represent the transfer load heuristic with target set and transfer load heuristic is decreases with increase in target set. There are total five delay graph with respect to the transfer load heuristic like Delay $6 \mathrm{hr}$, Delay $3 \mathrm{hr}$, Delay $2 \mathrm{hr}$, Delay $1 \mathrm{hr}$, and Delay0.5 hr. A target set of 0 to 3000 has been utilized and the transferred load varies from 1500 to 16000 . Diffent time frames have also been used in order to validate the results. Figure clearly represent that with in half an hour it transfer maximum amount of load and with in 6 hours it only transfered $10 \%$ of load.

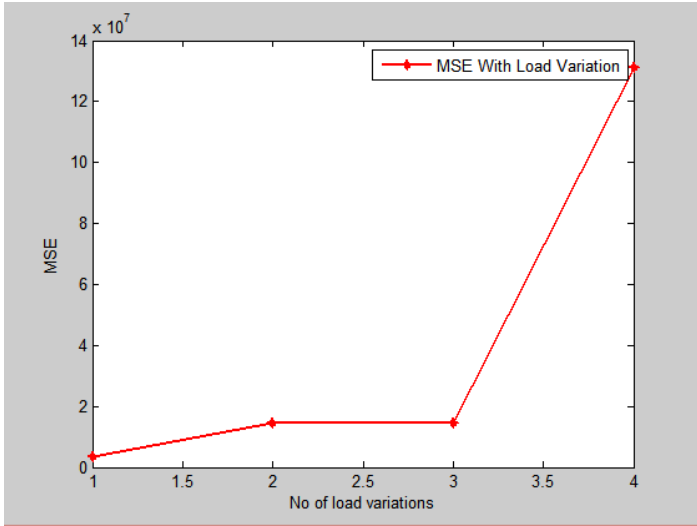

Figure 3: MSE with load variations

The above figure stands for different load variations and the deviation of the mean square error. In the figure the $\mathrm{x}$ axis denote the number of load variations and y axis denote MSE value. Above mentioned figure represent the obtained MSE with respect to the number of load variations and the MSE is increases with increase in the number of load variations. The figure depicts that at $1^{\text {st }}$ load varition it start increasing gradually till the $3^{\text {rd }}$ load variation but when it reaches $3^{\text {rd }}$ load variation the mean square error stars accumulative very fastly. This is because at the $3^{\text {rd }}$ load variation some noisy node get added in it. Due to which it intensification so fastly. The point where mean square error will rise, the Signal to noise ratio will be lowered.

Table 3: Mean Square Error

\begin{tabular}{|l|l|l|l|l|}
\hline MSE & 0.47 & 1.62 & 1.54 & $14 \times 10^{7}$ \\
\hline
\end{tabular}

The maximum value of MSE is near $14 \times 10^{7}$ and the minimum MSE is just near about the 1.

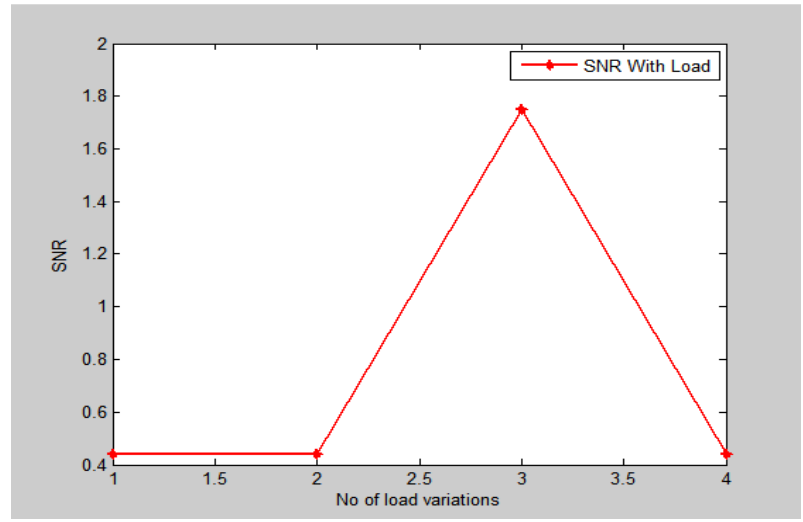

Figure 4: SNR With load variations

In the figure the $\mathrm{x}$ axis denote the number of load variations and y axis denote SNR value. No of load variations from 1 to 4 has been utilized and SNR varies from 0.4 to 2 . SNR is constant till $2^{\text {nd }}$ load variation because laod is added slowly but after $2^{\text {nd }}$ load variation it is increasing fastly and at $3^{\text {rd }}$ load variation it start decreasing because extra noisy nodes start adding in them. As mentioned in the above figure, mean square error is high at the third iteration and after the thrird iteration the SNR starts decresing. There are the range of the number of load variations is vary from 1 to 4 . 
Table 4: Signal Noise Ratio

\begin{tabular}{|l|l|l|l|l|}
\hline SNR & 0.43 & 0.43 & 1.79 & 0.43 \\
\hline
\end{tabular}

We obtained the maximum SNR 1.79 for the $3^{\text {rd }}$ load and the minimum load is observed for the 1 and 2 load. When compared battery consumption with existing approach these results are obtained and comparison in made between these two results.

Table 5: Battery Consumption Comparison

\begin{tabular}{|l|l|l|l|l|l|}
\hline Battery & 89.6 & 77.56 & 75.955 & 90.7 & 88.832 \\
$\begin{array}{l}\text { Consumption } \\
\text { Previous Work }\end{array}$ & 7536 & 435 & 46 & 656 & 48 \\
& & & & & \\
\hline $\begin{array}{l}\text { Battery } \\
\text { Consumption } \\
\begin{array}{l}\text { Proposed } \\
\text { Work }\end{array}\end{array}$ & 40.3 & 70.43 & 70.492 & 70.5 & 70.479 \\
& 231 & 82 & 317 & 21 \\
& & & & 4 & \\
\hline
\end{tabular}

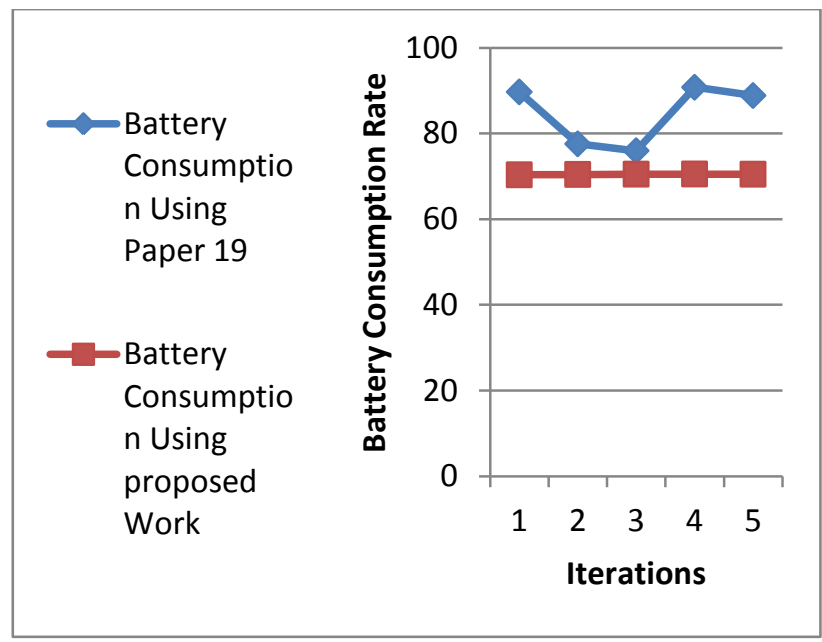

Figure 5: Battery Consumption comparison

Following results are obtained when the proposed work is compared with the existing work by means of transfer load delay. It is examined that the results obtained for proposed work are better than the previous one.

Table 6: Transfer Load Delay Comparison

\begin{tabular}{|l|l|l|l|l|l|}
\hline $\begin{array}{l}\text { Transfer Load In } \\
\text { Previous Work }\end{array}$ & $\begin{array}{l}4456.4 \\
524\end{array}$ & $\begin{array}{l}5938 . \\
4923\end{array}$ & $\begin{array}{l}4682 . \\
7345\end{array}$ & $\begin{array}{l}4213 . \\
8638\end{array}$ & $\begin{array}{l}3286 . \\
4823\end{array}$ \\
\hline $\begin{array}{l}\text { Transfer Load In } \\
\text { Proposed Work }\end{array}$ & 1219.4 & 1045. & $\begin{array}{l}871.0 \\
437\end{array}$ & $\begin{array}{l}696.8 \\
345\end{array}$ & $\begin{array}{l}522.6 \\
259\end{array}$ \\
\hline
\end{tabular}

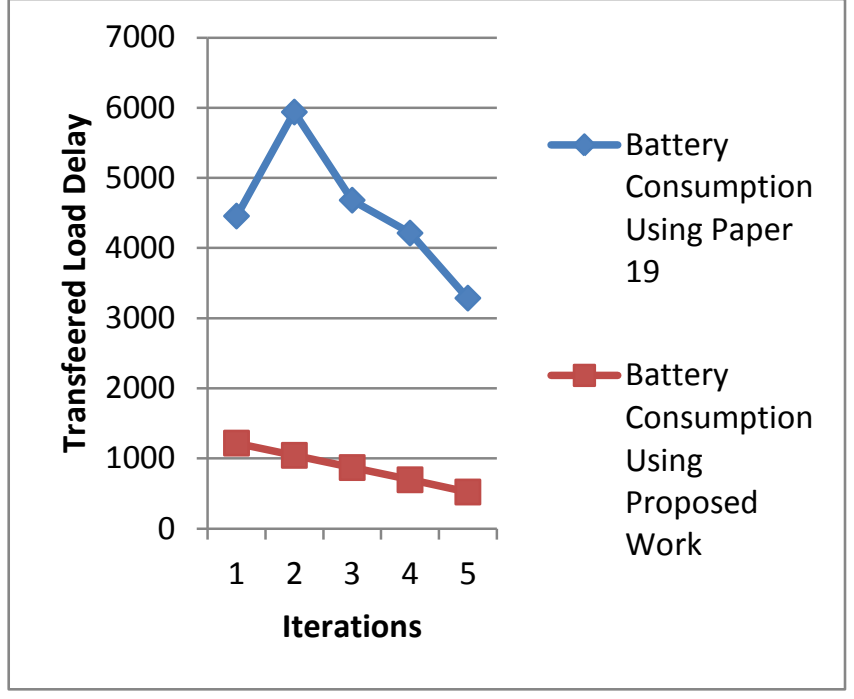

Figure 6: Transfer Load Delay Comparison

\section{CONCLUSION AND FUTURE WORK}

Social network is one of the biggest platform of performing where people share their views, photos, videos etc. Millions of people are connected to other millions of people. Mobile devices have become an easy way to access the social media account from anywhere. Misuse of the data present in the social network leads to draining the batteries of the network's user. In such a scenario there is a requirement to neutralize the group of the user so that mamximum battery power is attained with maximum of the utilization and communication strength . The proposed solution in the research work presents a unique idea to neutralize the network performance and to reduce the battery consumption of network users. The proposed solution has presented ways to group up the network and to set target nodes to the users. The proposed solution have also taken care of the normalization of the clusters as some noisy nodes has to be added in order to neutralize the group. The current research proposal opens a lot of gates for the future research workers. The future researach workers may try their hands in checking the network with more parameters like Delay , Communication error etc. The future aspect may also carry the usage of AI.

\section{REFERENCES}

[1] Cisco Visual networking index: Global mobile data traffic forecast update, 2015-2020," Cisco, San Jose, CA, USA, White Paper, Feb. 2013.

[2] K. Lee, I. Rhee, J. Lee, S. Chong, and Y. Yi, "Mobile data offloading: how much can Wi-Fi deliver" in Proceedings of the 6th International Conference, ser. Co-NEXT '10. New York, NY, USA: ACM, 2010, pp. 26:1-26:12.

[3] S. Dimatteo, P. Hui, B. Han, and V. Li, "Cellular traffic offloading through Wi-Fi networks," in Proc. IEEE MASS, Oct. 2011.

[4] K. Kumar, J. Liu, YH. Lu, "A survey of Computation Offloading for Mobile Systems," in Mobile Network Application, vol.18, no. 1,pp.129-140, Feb, 2013.

[5] F. Rebecchi, M. Amorim, V. Conan, A. Passarella, R Bruno, and M Conti, "Data Offloading Techniques in Cellular Networks: A Survey", IEEE Communication Surveys, vol. 17, pp. 580-603, 2015. 
[6] Aijaz, H. Aghvami, and M. Amani, "A survey on mobile data offloading: technical and business perspectives," IEEE Wireless Communications, vol. 20, pp. 104-112, April 2013

[7] Rahul Raj Kumar Pahlajani and Dr. G. R. Bamnote "Mobile Data Offloading the Growing Need with Its Solutions and Challenges," inIJRITCC, March 2014.

[8] Balasubramanian, R. Mahajan, and A. Venkataramani, "Augmenting Mobile 3G Using Wi-Fi," in ACM Mobile Sys 2010, Jun. 2010, pp. 209-222.

[9] N. Ristanovic, J. L. Boudec, A. Chaintreau, and V. Erramilli, "Energy efficient offloading of 3G networks," in Proc. 8th Int. IEEE Conf. MASS, Oct. 2011, pp. 202211.

[10] S. Singh, H. Dhillon, and J. Andrews, "Offloading in heterogeneous networks: Modeling, analysis, and design insights," IEEE Transactions on Wireless Communications, vol. 12, no. 5, pp. 2484-2497, May 2013.

[11] Alexander Pyattaevy, Kerstin Johnsson, Sergey Andreevy, and YevgeniKoucheryavyy"3GPP LTE traffic offloading onto Wi-Fi Direct," workshop on Mobile Internet IEEE 2013, pp 134-140.

[12] S. Sou, "Mobile data offloading with policy and charging control in 3GPP core network," IEEE Trans. Veh. Technol., vol. 62, no. 7, pp. 3481-3486, Sep. 2013.

[13] F. Mehmeti and T. Spyropoulos, "Performance analysis of on-the-spot mobile data offloading in heterogeneous networks," in IEEE GLOBECOM, 2013.

[14] X. Kang, Y. K. Chia, and S. Sun. "Mobile data offloading through a third-party Wi-Fi access point: An operator's perspective." IEEE Transactions on Wireless Communications, 13(10):5340-5351, 2014.

[15] DongeunSuh, HaneulKo, and Sangheon Pack, "Efficiency Analysis of Wi-Fi Offloading Techniques" IEEE Transactions On Vehicular Technology, vol. 65, no. 5, pp. 3813-3817, May 2016.

[16] G. Fortetsanakis and Maria Papadopouli, "How Beneficial is the Wi-Fi Offloading, A Detailed GameTheoretical Analysis in Wireless Oligopolies," World of Wireless, Mobile and Multimedia Networks (WoWMoM), 2016 IEEE 17th International Symposium on A.

[17] S. Ioannidis, A. Chaintreau, and L. Massouli'e, "Optimal and scalable distribution of content updates over a mobile social network," in IEEE INFOCOM, Rio de Janeiro, Brazil, Apr. 2009.

[18] Y. Li, G. Su, P. Hui, D. Jin, L. Su, and L. Zeng, "Multiple mobile data offloading through delay tolerant networks," in Proc. of the 6th ACM Workshop on Challenged Networks, ser. CHANTS '11. New York, NY, USA: ACM, 2011, pp. 43-48.

[19] Y. Chuang and K.-J. Lin, "Cellular traffic offloading through community-based opportunistic dissemination," in IEEE Wireless Communications and Networking Conference (WCNC), Shanghai, China, Apr. 2012, pp. 3188-3193.

[20] B. Han, P. Hui, V. Kumar, M. Marathe, J. Shao, and A. Srinivasan, "Mobile Data Offloading through Opportunistic Communications and Social
Participation," IEEE Trans. Mobile Computing, vol. 11, no. 5, pp. 821-834, May 2012.

[21] P. Baier and K. Rothermel, "TOMP: Opportunistic traffic offloading using movement predictions," in IEEE Conference on Local Computer Networks (LCN), Oct. 2012.

[22] L. Valerio, R. Bruno, and A. Passarella, "Adaptive data offloading in opportunistic networks through an actorcritic learning method," in ACM CHANTS, Maui, HI, Sep. 2014.

[23] M. V. Barbera, A. C. Viana, M. D. de Amorim, and J. Stefa, "Data offloading in social mobile networks through VIP delegation," Ad Hoc Networks, vol. 19, pp. 92-110, 2014.

[24] X. Wang, M. Chen, Z. Han, D. Wu, and T. Kwon, "Toss: traffic offloading by social network service-based opportunistic sharing in mobile social networks," In IEEE INFOCOM, 2014

[25] M. Xiao, J. Wu, H. Huang, L. Huang, and C. Hu, "Deadline-Sensitive User Recruitment for Probabilistically Collaborative Mobile Crowdsensing," Proc. of the 36th IEEE International Conference on Distributed Computing Systems (ICDCS 2016), June 27 30, 2016.

[26] X. Zhuo, W. Gao, G. Cao, and Y. Dai, "Win-Coupon: An Incentive Framework for 3G Traffic Offloading," Proc. IEEE Int'l Conf. Network Protocols (ICNP), 2011.

[27] J. Lee, Y. Yi, S. Chong, and Y. Jin, "Economics of Wi-Fi offloading: Trading delay for cellular capacity," IEEE Trans. Wireless Commun., vol. 13, no. 3, pp. 1540-1554, Mar. 2014.

[28] X. Zhuo, W. Gao, G. Cao, and S. Hua, "An incentive framework for cellular traffic offloading," IEEE Trans. Mobile Comput., vol. 13, no. 3, pp. 541-555, Mar. 2014.

[29] B. Jung, N. Song, and D. Sung, "A network-assisted user-centric Wi-Fi offloading model for maximizing peruser throughput in a heterogeneous network," IEEE Trans. Veh. Technol., vol. 63, no. 4, pp. 1940-1945 May 2014.

[30] L. Gao, G. Iosifidis, J. Huang, L. Tassiulas, and D. Li, "Bargaining-based mobile data offloading," IEEE J. Sel. Areas Commun., vol. 32, no. 6, pp. 1114-1125, Jun. 2014.

[31] G. Iosifidis, L. Gao, J. Huang, and L. Tassiulas, "A double-auction mechanism for mobile data-offloading markets," IEEE/ACM Transactions on Networking, vol. 23 , no. 5,2015

[32] http://www.strategies.nzl.com/wpapers/2012013.htm

[33] Wenchao Xu, Yuan Wu, HaiboZhou,Yuanguo Bi, Nan Cheng, Xuemin(Sherman) Shen,"Wi-Fi: Terminal-toTerminal Communication incorporated Wi-Fi Offloading," 2016 IEEE

[34] Y. Khadraoui, X. Lagrange, A. Gravey, "A Survey of Available Features for Mobile Traffic Offload," in proceeding of European Wireless 2014,Barcelona 2014. 